\title{
USO DE SISTEMAS AUTOMATIZADOS \\ EN LAS BIBLIOTECAS UNIVERSITARIAS ESPAÑOLAS
}

\author{
Purificación Moscoso*, Yolanda Ríos"*
}

Resumen: Se estudia el uso de los sistemas de automatización en las bibliotecas universitarias españolas. Se elabora un estudio estadístico con datos sobre los soportes fisicos y softwares utilizados, módulos en funcionamiento, formatos de entrada y salida, asi como fuentes y mecanismos para la carga de registros en el sistema, estado y métodos de la conversión retrospectiva, uso de nuevas tecnologias, y nuevos servicios implantados desde 1986 en este tipo de bibliotecas. Posteriormente, se analizan los resultados obtenidos y se comparan con datos de 1986.

Palabras clave: bibliotecas universitarias, sistemas automatizados.

Abstract: This article studies the use of automated systems in Spanish academic libraries. A statistical analysis is done about the hardware and software used, installed modules, input and output formats, sources and mechanisms for downloading records, state of the art and methods of the retrospective conversion, use of new technologies and new services introduced since 1986. Once the data are analysed, they are compared with data obtained in a similar study carried out in 1986.

Keywords: academic libraries, automated systems.

\section{Introducción}

A petición de la Comisión de las Comunidades Europeas se ha llevado a cabo un estudio sobre el uso de sistemas automatizados en las bibliotecas españolas. Este estudio es la actualización del trabajo, realizado en 1986, Estado Actual de las Nuevas Tecnologías de la Información en las Bibliotecas y su Impacto sobre el Funcionamiento Bibliotecario: España (1) (más comúnmente conocido con el nombre de $L I B-2)$.

El objetivo de esta puesta al día de datos es obtener información sobre el uso de las nuevas tecnologías de la información en las bibliotecas españolas, para identificar los cambios significativos acaecidos desde 1986, así como las tendencias dominantes.

En este artículo se analizan los resultados relativos a las bibliotecas universitarias.

Primeramente se elabora un estudio estadístico sobre los soportes físicos y programas utilizados, módulos en funcionamiento, formatos de entrada y salida, así como las fuentes y mecanismos para la carga de registros en el sistema, el estado y métodos de la conversión retrospectiva, uso de nuevas tecnologías y servicios introducidos desde 1986 en este tipo de bibliotecas. Se proporciona también información sobre las redes informáticas utilizadas en este entorno.

Una vez realizado el estudio estadístico se procede a un análisis cualitativo de

- Diplomatura de Biblioteconomía y Documentación. Universidad Carlos III de Madrid.

* Unidad de Coordinación de Bibliotecas. CSIC.

Recibido 16-5-91 
los resultados obtenidos, y se compara con datos del primer estudio, llevado a cabo en el año 1986.

Por último, se proporciona también información comparativa con respecto a otros tipos de bibliotecas, especialmente públicas y especializadas.

Es importante señalar que, para este estudio, se ha centralizado la información relativa a las bibliotecas automatizadas de las distintas facultades en las bibliotecas generales de cada universidad. Cuando la biblioteca general no estaba automatizada, pero sí la de alguna facultad, se han tenido en cuenta los datos relativos a ésta. Tal es el caso, por ejemplo, de la facultad de Derecho de la Universidad de Alicante.

También es necesario aclarar que el criterio seguido para considerar que una biblioteca está automatizada es que tenga un software en funcionamiento y que cuente con registros automatizados en su catálogo.

\section{Metodología}

Para la identificación de las universidades con bibliotecas automatizadas se utilizó información ya disponible en la Unidad de Coordinación de Bibliotecas del Consejo Superior de Investigaciones Científicas.

De 39 universidades españolas, 16 están automatizadas, lo que supone un porcentaje de automatización en este tipo de bibliotecas del $41 \%$.

Una vez identificadas las universidades con bibliotecas informatizadas se envió un cuestionario a las mismas, en el que se solicitaba información sobre los fondos de la biblioteca, equipos y sistemas informáticos utilizados, costes de la automatización, módulos en funcionamiento, número de registros en soporte magnético, formato informático de los registros, normas de catalogación usadas, fuentes y mecanismos para la carga de registros en el sistema, estado y métodos de la conversión retrospectiva, y nuevos servicios introducidos desde 1986.

El cuestionario se envió a principios del mes de octubre de 1990, con la idea de limitar el estudio a aquéllos recibidos antes del 31 de diciembre de ese mismo año. Sin embargo, la falta de respuestas obtenidas hizo necesaria la ampliación del plazo hasta finales de febrero de 1991. Así, los datos incluidos en este artículo se refieren sólo hasta la fecha señalada. Llamamos la atención sobre este hecho puesto que las cifras en el tipo de información de la que se está hablando varian con gran rapidez. Por lo tanto, al referirnos, por ejemplo, al número de registros automatizados de cada centro, es importante tener en cuenta que para la fecha de publicación del artículo esta cifra habrá sido sobrepasada.

De 16 encuestas enviadas contestaron 15, lo que supone un $94 \%$ de nivel de respuesta. Por lo tanto, la información que se proporciona excluye una universidad, debido a que ésta no contestó al cuestionario enviado.

La verificación de la información obtenida y la aclaración de algunos puntos del cuestionario se realizaron mediante llamadas telefónicas a los responsables directos de la automatización de estos centros.

Para el análisis estadístico de los datos se elaboró una hoja electrónica con el programa LOTUS 123 , versión 3.0 , en la que se incluía la información recopilada mediante los cuestionarios. 
Una vez obtenida la información estadística se procedió a su análisis cualitativo, para finalmente comparar los resultados relativos a este período con los obtenidos en el primer estudio realizado en 1986.

\section{Análisis estadístico de los resultados}

De 39 universidades españolas, 16 tienen sistemas automatizados en funcionamiento, lo que supone un porcentaje de automatización del $41 \%$ en este tipo de bibliotecas.

El número total de registros en soporte magnético de las universidades españolas es 757.333 , de los cuales 697.732 corresponden a monografias, y 59.601 a publicaciones periódicas. En la tabla 1 se ofrece una relación del número de registros de cada centro universitario, acompañada del tipo de formato utilizado y en la tabla 2 se relaciona el número de registros con el software utilizado.

Hay que indicar que las cifras referentes a números totales de registros incluyen un número de duplicados imposible de conocer al no existir catálogos colectivos.

\section{Tabla 1}

Bibliotecas universitarias. Número de registros por formato de salida

\begin{tabular}{lrc}
\hline & Monografias & $\begin{array}{c}\text { Publicaciones } \\
\text { periódicas }\end{array}$ \\
\hline IBERMARC & 17.582 & \\
Politécnica de Cataluña & 130.000 & 4.210 \\
Politécnica de Valencia & 6.300 & 1.398 \\
Carlos III de Madrid & 28.100 & \\
Cantabria & 10.100 & 4.300 \\
Alicante & 192.082 & 9.908 \\
$\quad$ Total & & \\
DMARC & 10.500 & \\
Deusto & 50.000 & \\
Granada & 12.000 & \\
Politécnica de Madrid & 400 & \\
Valencia & 10.000 & 11.273 \\
Sevilla & 117.298 & \\
Navarra & 15.000 & \\
UNED & 1.308 & \\
Zaragoza & 82.920 & \\
Oviedo & 299.426 & \\
$\quad$ Total & & \\
CATMARC & 17.582 & 4.210 \\
Politécnica de Cataluña & 171.060 & 30.000 \\
Barcelona & 188.642 & \\
Total & & 4.210 \\
UNIMARC & 17.582 & \\
Politécnica de Cataluña & & \\
\hline
\end{tabular}


Tabla 2

Bibliotecas universitarias. Número de registros por software

\begin{tabular}{lrc}
\hline & Monografias & $\begin{array}{c}\text { Publicaciones } \\
\text { periódicas }\end{array}$ \\
\hline DOBIS/LIBIS & & \\
Deusto & 10.500 & \\
Granada & 50.000 & \\
Politécnica de Madrid & 12.000 & \\
Valencia & 400 & \\
Sevilla & 10.000 & \\
Navarra & 117.298 & 11.273 \\
UNED & 15.000 & \\
Zaragoza & 1.308 & \\
Oviedo & 82.920 & \\
$\quad$ Total & 299.426 & 11.273 \\
SABINI & & \\
Cantabria & 28.100 & \\
Alicante & 10.100 & 4.300 \\
$\quad$ Total & 38.200 & 4.300 \\
VTLS & & \\
Politécnica de Cataluna & 17.582 & 4.210 \\
ABSYS & & \\
Carlos III & 6.300 & \\
AD HOC & & \\
Barcelona & 171.060 & 30.000 \\
Politécnica de Valencia & 130.000 & 1.398 \\
$\quad$ Total & 301.060 & 31.398 \\
$\quad$ Total registros & 662.568 & 51.181 \\
\hline$\quad$ & & \\
\hline
\end{tabular}

Los equipos utilizados para la automatización de las bibliotecas universitarias son, en su mayoría, grandes ordenadores. Un 58,8\% de estas bibliotecas los utiliza, frente a un $23,5 \%$ que hace uso de mini ordenadores, y un $27,6 \%$ de microordenadores (figura 1).

El $66,66 \%$ comparte el ordenador con otros departamentos, mientras que el $33,33 \%$ no lo hace.

El software más usado para la automatización de las bibliotecas universitarias es el DOBIS/LIBIS. Este sistema lo usa un $60 \%$ de las mismas. El resto de los softwares utilizados son SABINI, ABSYS y VTLS. SABINI está en funcionamiento en el $13,33 \%$ de estos centros, ABSYS, en el $6,67 \%$ y VTLS, también en el $6,67 \%$.

Con respecto a otros tipos de bibliotecas, se observa que el DOBIS/LIBIS ocupa también un lugar importante en las bibliotecas públicas. Este sistema está instalado en el $23,33 \%$ de éstas, mientras que no tiene presencia alguna en las bibliotecas especializadas. Los sistemas comerciales predominantes en las públicas son el SABINI y el PALS (2), utilizados, cada uno, en el $26,67 \%$ de estos centros. 
Figura 1

Porcentaje de bibliotecas universitarias según el hardware utilizado

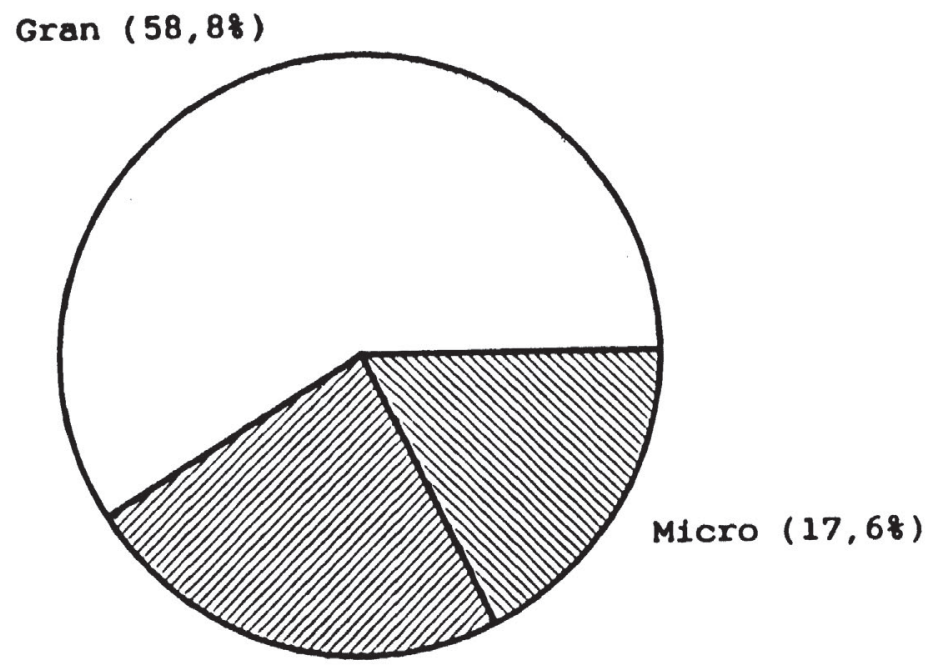

$\operatorname{Min} 1(23,58)$

En las especializadas el SABINI está en funcionamiento en el $22,5 \%$ de los casos, y ABSYS en el $2,5 \%$.

La figura 2 muestra una comparación del uso de los sistemas comerciales en los distintos tipos de bibliotecas.

En cuanto a los programas diseñados «ad hoc» hay que resaltar que la tendencia es a su no utilización. Sólo dos bibliotecas universitarias utilizan en la actualidad un programa diseñado «ad hoc», es decir, un 13,33\%. Esta tendencia a la no utilización de programas "a medida» se manifiesta también en las bibliotecas públicas, y contrasta con las especializadas, en las que el uso de este tipo de programas predomina, frente a la utilización de sistemas comerciales. El porcentaje de utilización de programas "ad hoc» en las bibliotecas especializadas es del $42,5 \%$; el mayor con respecto a otros tipos de bibliotecas. Sólo un $10 \%$ de las bibliotecas públicas tienen un sistema «ad hoc» instalado.

En cuanto a los módulos en funcionamiento en las bibliotecas universitarias es el de catalogación el más utilizado. El $100 \%$ de estas bibliotecas lo tienen en funcionamiento. El porcentaje de bibliotecas universitarias que tienen automatizado el catálogo de acceso público es también muy elevado. El 86,67\% de éstas dicen tenerlo en funcionamiento. Las adquisiciones están en funcionamiento en el $53,33 \%$ de las universidades, el módulo de préstamo en el 33,33\%, las suscripciones en el $20 \%$, y el préstamo interbibliotecario sólo está informatizado en el $6,67 \%$ de los casos.

En la figura 3 se muestran los porcentajes de cada tipo de biblioteca según los módulos instalados.

Con respecto a los formatos de entrada y salida, la totalidad de las universidades españolas utiliza uno de tipo MARC. Como ya se ha dicho, en la tabla 1 se ofrece una relación del tipo de formato usado, acompañada del número de 
Figura 2

Porcentaje de bibliotecas según su tipo y el software utilizado

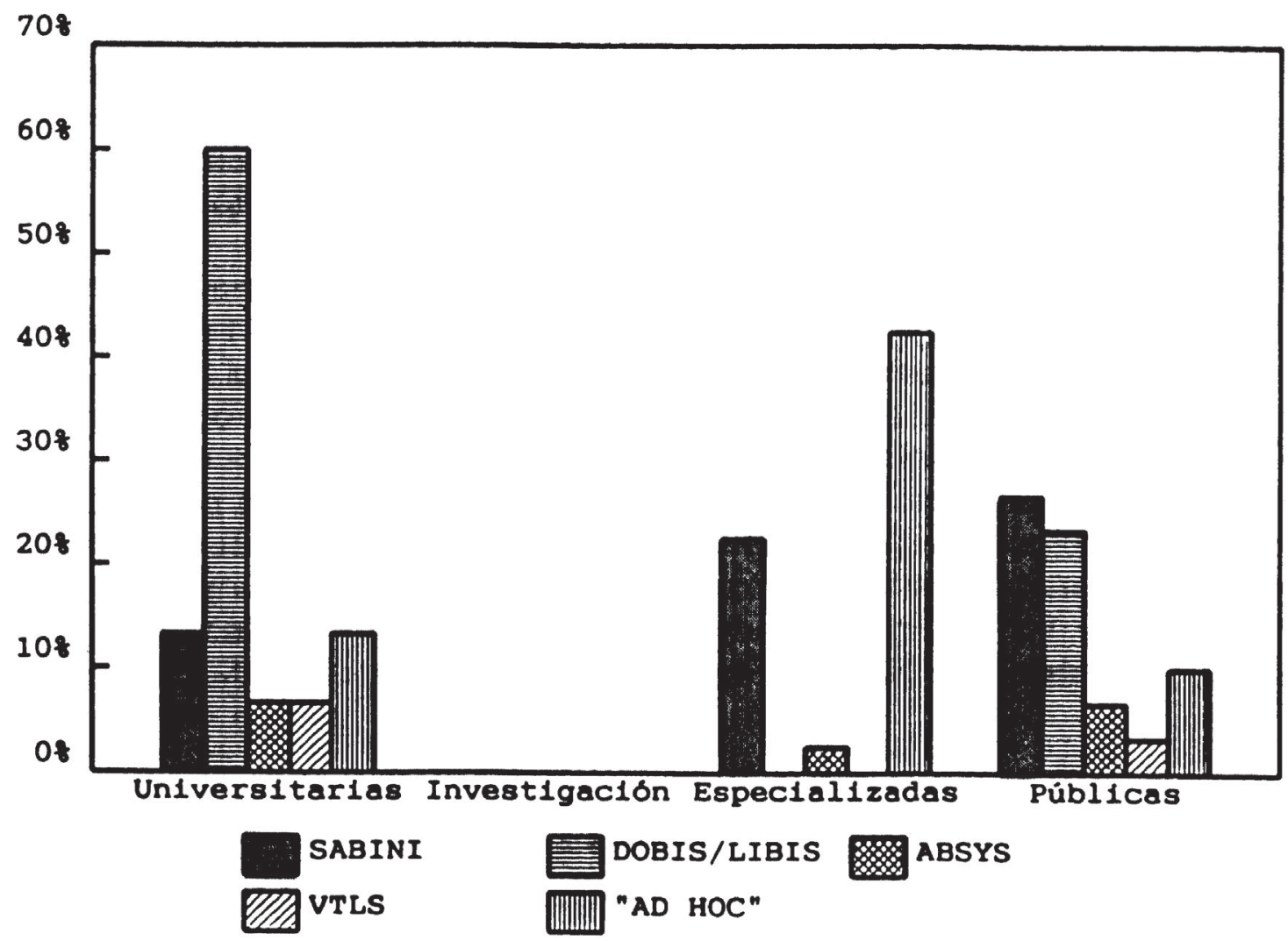

Figura 3

Porcentaje de bibliotecas según su tipo y los módulos en funcionamiento

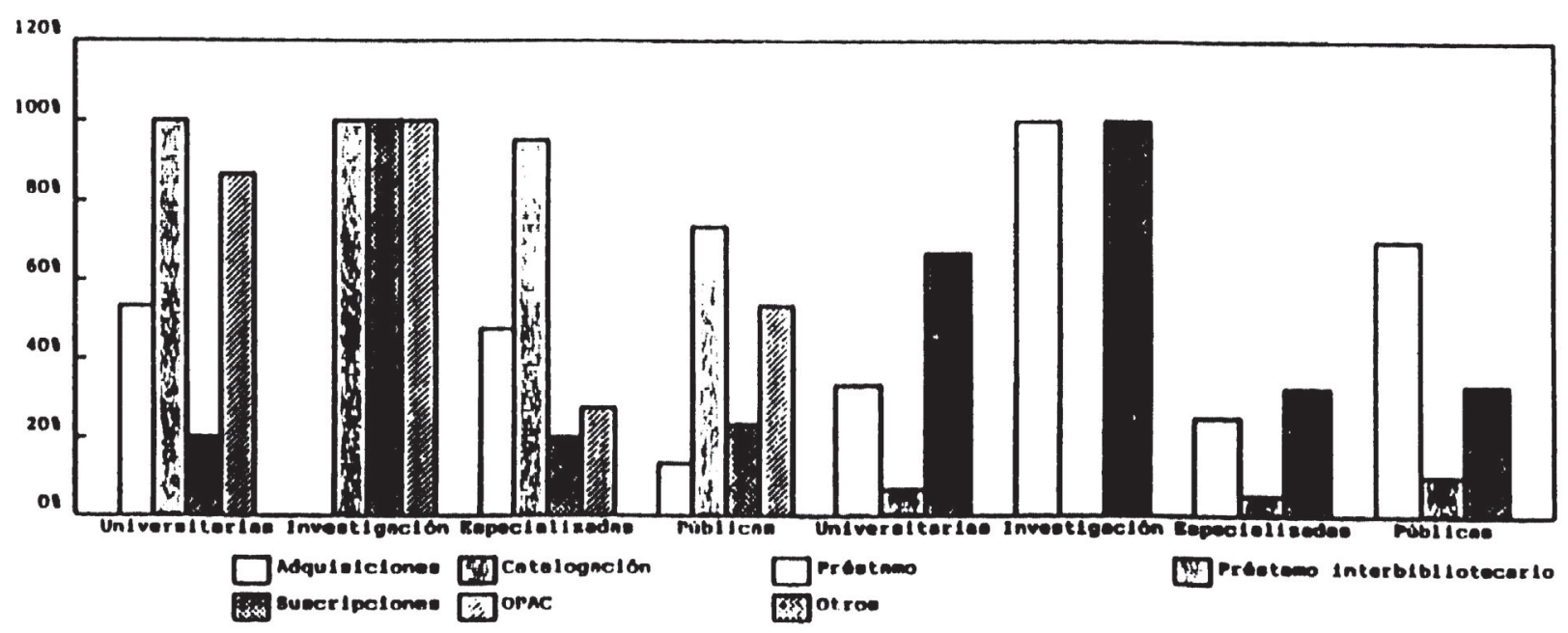


registros automatizados en cada centro universitario (relación en 1991). La utilización de un formato de tipo MARC es una tendencia generalizada en las bibliotecas españolas, con excepción de las bibliotecas del Consejo Superior de Investigaciones Científicas (que utilizan un formato definido expresamente para ellas), y las bibliotecas especializadas, que son el tipo de biblioteca que más hace uso de formatos de definición propia.

Las fuentes que se han identificado para la carga de registros en el sistema son el uso de bases de datos, tanto en línea como en CD-ROM, bibliografias, y otros catálogos de bibliotecas.

De todas las bibliotecas automatizadas, son las universitarias las que más utilizan como fuente de carga las bases de datos. Estas se usan en el $50 \%$ de este tipo de bibliotecas. En cuanto al uso de otros tipos de fuentes, el 14,29\% utiliza bibliografias, y sólo el $7,14 \%$ recurre a otros catálogos de bibliotecas.

En la figura 4 se muestra el uso de los distintos tipos de fuentes en los distintos tipos de bibliotecas.

En lo que respecta a los mecanismos de carga de registros, un $20 \%$ de las bibliotecas universitarias utiliza el volcado de registro por registro, y un 13,33\% la transferencia de ficheros. El volcado de registros es el método utilizado en el $33,33 \%$ de las bibliotecas públicas, y en el $25 \%$ de las especializadas; mientras que

Figura 4

Porcentaje de bibliotecas según su tipo y las fuentes de carga de registros

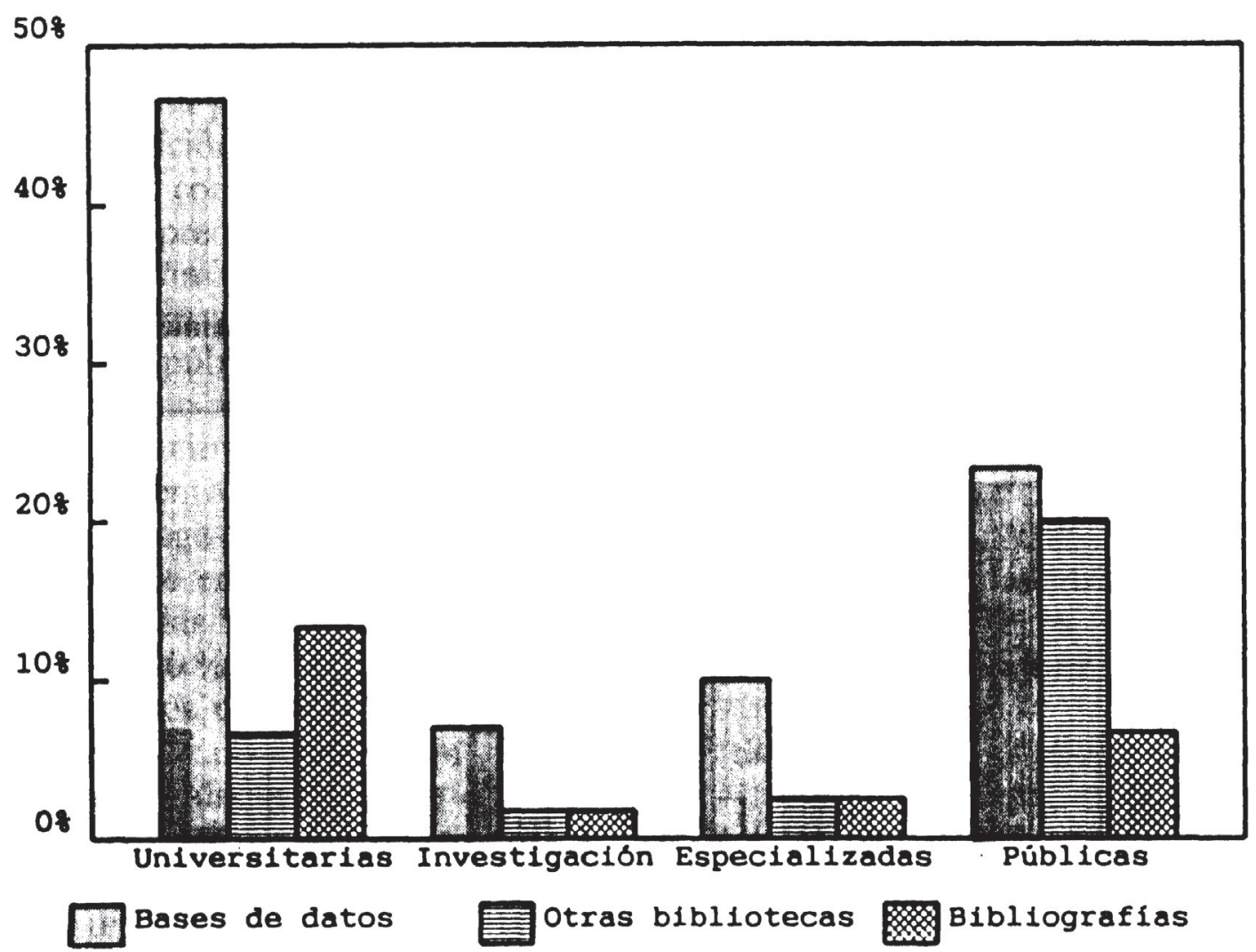


la transferencia de ficheros se utiliza en un $46,67 \%$ de las públicas y en un $16 \%$ de las especializadas (figura 5).

Son las bibliotecas universitarias las que más atraso llevan en el proceso de conversión retrospectiva. Aunque este proceso está en marcha en el $60 \%$ de estos centros, sólo está terminado en el $6,67 \%$ de los casos. Un $26,67 \%$ no lo ha comenzado todavía.

El método predominante para la realización de la retroconversión es el de grabación en la propia biblioteca, utilizado por el $53,33 \%$ de las bibliotecas universitarias. El $20 \%$ de éstas ha recurrido al uso de empresas de servicios, y también un $20 \%$ a la utilización de bases de datos, tanto en línea como en CDROM.

En las figuras 6 y 7 se muestra el estado de la retroconversión en los distintos tipos de bibliotecas, así como los métodos utilizados para ella.

El uso de nuevas tecnologías en las universidades españolas se limita al ącceso a bases de datos en discos CD-ROM. Un $80 \%$ de las universidades tienen acceso a este servicio (3). Las bases de datos en CD-ROM se usan, en las bibliotecas universitarias, principalmente, para llevar a cabo búsquedas bibliográficas $(73,33 \%)$, para la catalogación y adquisición de ejemplares $(46,66 \%$ y $40 \%$, respectivamente), para la conversión retrospectiva $(33,33 \%)$ y para el préstamo interbibliotecario $(20 \%)$.

\section{Figura 5}

Porcentaje de bibliotecas según su tipo y los mecanismos de carga de registros

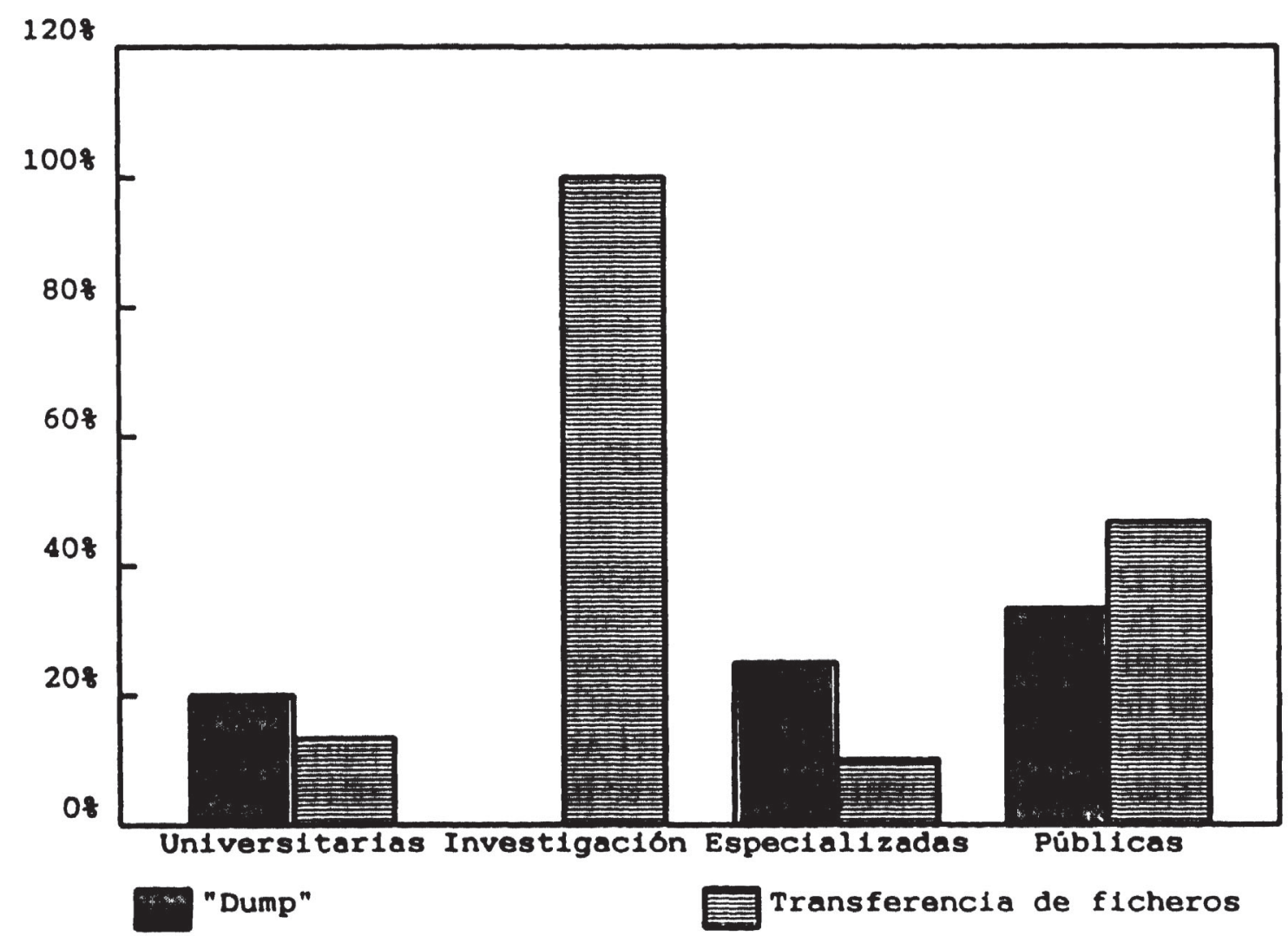


Figura 6

Porcentaje de bibliotecas según su tipo y la situación de la conversión retrospectiva

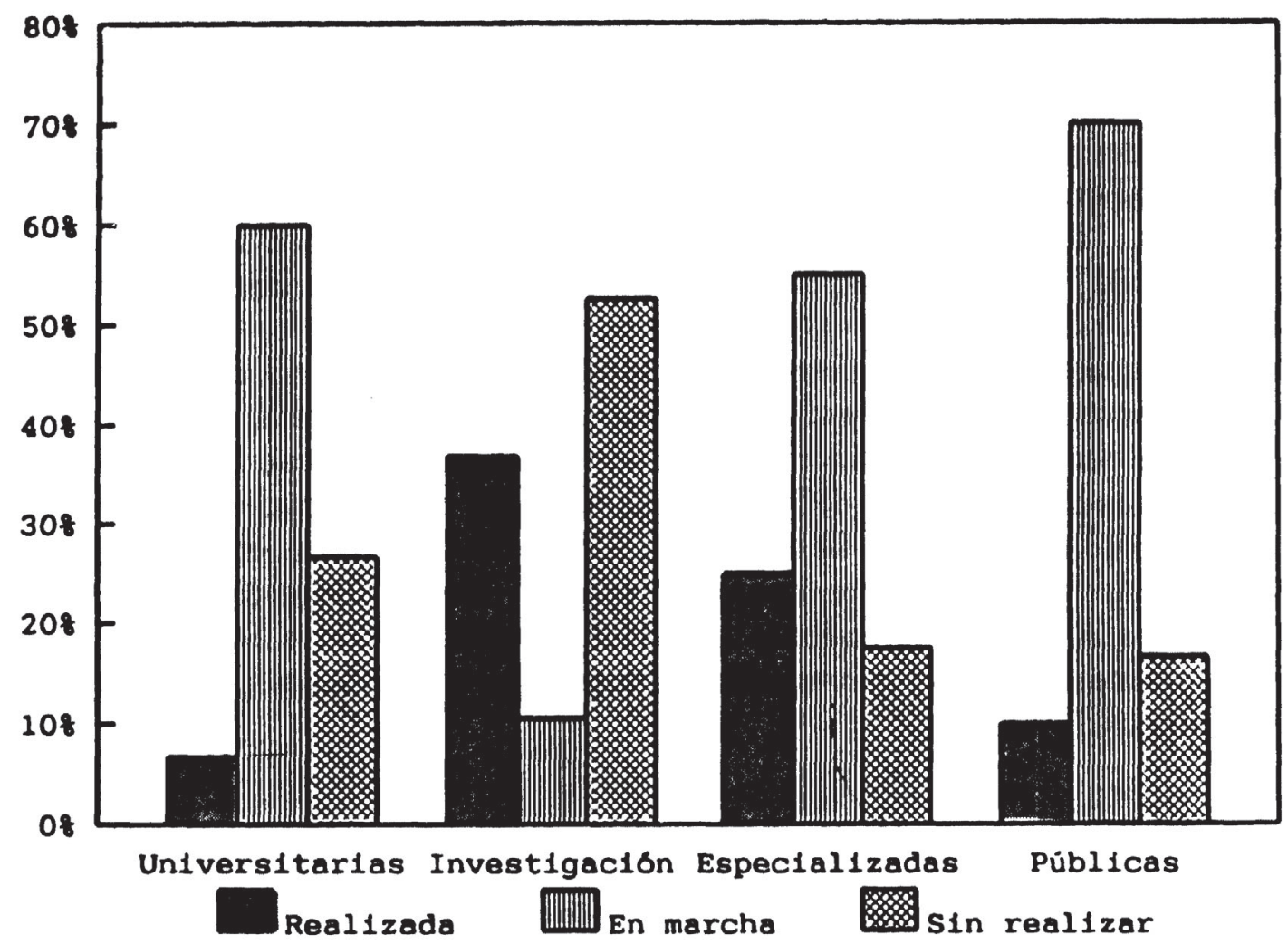

Otros nuevos servicios introducidos desde esta fecha son el fax $(73,33 \%)$, acceso a bases de datos en línea $(46,67 \%)$, correo electrónico $(40 \%)$, petición automatizada de documentos primarios $(33,33 \%)$, acceso a catálogos distintos al de la propia biblioteca $(33,33 \%)$, y videotex $(6,67 \%)$.

\section{Redes Informatizadas}

En cuanto a las redes informatizadas, es importante destacar el uso de la red DOBIS/LIBIS por parte de aquellas universidades cuyas bibliotecas están automatizadas con este sistema.

La red DOBIS/LIBIS surgió hace poco más de un año a raíz del plan de acción de la CEE. Consta de un nodo central ubicado en la Universidad de Oviedo, en el cual existe un catálogo colectivo con toda la información bibliográfica de cada una de las instalaciones. El acceso al catálogo se realiza mediante líneas x.25 de la red pública de transporte IBERPAC, con una velocidad de transmisión de 4.800 bps. En el nodo central, además, se ha instalado el software de comunicaciones necesario para que, si asi lo desean, cada institución pueda acceder, además de al catálogo bibliográfico propio, al de cada uno de los integrantes de la red. La conexión al nodo central podrá realizarse de varias formas: conexión host-host, vía 
Figura 7

Porcentaje de bibliotecas segán su tipo y los métodos de conversión retrospectiva

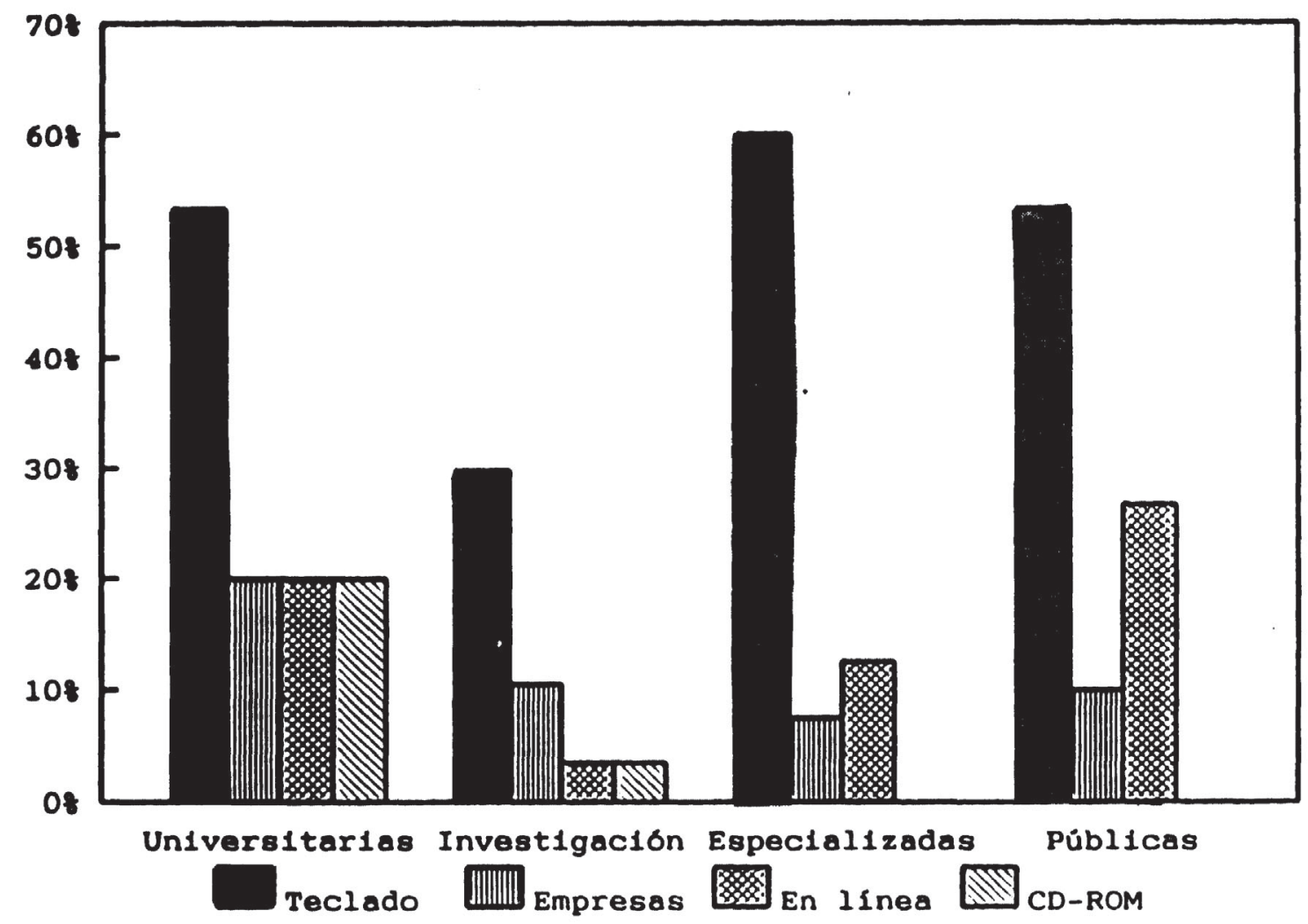

ordenadores dotados de las oportunas tarjetas para comunicaciones $\mathrm{x} .25$, via redes locales, etc.

Los objetivos de la red son:

- Creación de un catálogo colectivo acumulado, que es el núcleo central integrado de las bibliotecas.

- Captación e intercambio de registros con el fin de reducir tiempos y costes de catalogación.

- Hacer extensivas las funciones de interconexión y captación de información a nivel europeo.

Las universidades que forman parte de la red son: Deusto, Granada, Navarra, Oviedo, Salamanca, Sevilla y UNED.

Además de esta red, próximamente entrará en funcionamiento la Red de Bibliotecas Universitarias (REBIUN). Su objetivo es la cooperación en todos los campos de trabajo e intereses de las bibliotecas universitarias. Por esta razón, en principio, está restringida a bibliotecas universitarias. Actualmente se están abordando los siguientes temas: Préstamo interbibliotecario, catálogos colectivos/ catalogación cooperativa, formación de plantillas, y reglamentos de Régimen Interior de Bibliotecas Universitarias. 
Componen la red nueve universidades: Alcalá de Henares, Barcelona, Cantabria, Oviedo, País Vasco, Politécnica de Cataluña, Santiago de Compostela, Sevilla y UNED. Con el anuncio oficial de la red se informará de los modos de ingresar, participar o colaborar en/o con la red. En principio, se pretende que al menos sea posible: ser miembro de la red, suscribir acuerdos vigentes en la red sobre aspectos concretos, y firmar convenios con la red.

\section{Análisis de los datos}

La mayoritaria utilización de grandes ordenadores en las bibliotecas universitarias responde, por un lado, a las necesidades de estos centros, y, por otro, al hecho de tratarse, en la mayoría de los casos, de equipos compartidos con otros departamentos. Así, la necesidad de procesar grandes volúmenes de información hace necesaria la automatización con ordenadores de gran capacidad. Además, la tendencia a compartir equipos hace más propicia la automatización con este tipo de ordenadores.

Aun cuando, en principio, el hecho de compartir el ordenador con departamentos ajenos a la biblioteca es más proclive a plantear cierto tipo de problemas, en estos casos no parece haber supuesto desventajas significativas en el desarrollo de la mecanización.

La principal razón que hace del DOBIS/LIBIS el programa mayoritariamente utilizado en las bibliotecas de las universidades españolas es que un gran número de estas instituciones contaba ya con ordenadores IBM para el desarrollo de algunas de las funciones de la universidad. Al ser la tendencia que la biblioteca, para su mecanización, hiciera uso de las instalaciones informáticas ya existentes, era necesario, pues, elegir un sistema que corriera en este tipo de ordenadores. De los programas existentes en el mercado español en los orígenes de la automatización de estos centros, era el DOBIS/LIBIS el que mejor se adaptaba a estas especificaciones.

Es importante recalcar que la oferta de programas comerciales ha crecido considerablemente desde 1986. Así, mientras que en este año la oferta se limitaba al SABINI, DOBIS/LIBIS y ALEPH, hoy ésta se ha ampliado con la introducción de ABSYS, PALS y VTLS, además del sistema desarrollado para la Biblioteca Nacional, ILIADA - que nace con clara vocación comercial- y LIBERTAS, de próxima instalación en alguna biblioteca universitaria.

Ahora bien, pese a que la lista de softwares comerciales ha crecido considerablemente, las universidades han seguido decantándose, en su mayoría, hacia la instalación del DOBIS/LIBIS (cuatro universidades en 1986, nueve en este momento). El SABINI no ha parecido dar buenos resultados en grandes centros, por lo que de cuatro universidades que tenian intención de instalarlo en 1986, son sólo hoy dos las que lo utilizan. Es de suponer, sin embargo, que el aumento en la variedad de softwares comerciales repercuta, en un plazo más o menos largo, en la variedad de softwares utilizados, tanto en las bibliotecas universitarias como en los otros tipos de bibliotecas.

Un cambio muy significativo ha sido el progresivo abandono de los intentos de automatización con programas «ad hoc» en las bibliotecas universitarias, desarro- 
llados por sus centros de cálculo. La idea de automatizar con programas diseñados «a medida» era generalizada en 1986, pero el hecho comprobado de que este tipo de programas pueden llegar a generar más problemas que el uso de sistemas comerciales ha resultado en el ya mencionado abandono de esta idea. Así, encontramos que de 10 universidades que planeaban instalar sistemas de este tipo en 1986, hoy son sólo dos las que los utilizan.

En cuanto a los formatos de entrada, la utilización de uno de tipo MARC es el resultado del interés por el intercambio y la cooperación, no sólo con respecto a las bibliotecas españolas, sino también con las de otros países, ya que los formatos de tipo MARC son los más utilizados en el mundo occidental.

El cambio fundamental relativo al tipo de formato utilizado con respecto al panorama de 1986 ha sido el aumento del número de bibliotecas universitarias que usan el DMARC (como resultado de la instalación de DOBIS/LIBIS). Asi, tres universidades disponían de IBERMARC, tres de CATMARC, una de DMARC y una también de UNIMARC. Hoy son nueve las que utilizan DMARC, seis IBERMARC, tres CATMARC y una UNIMARC.

En cuanto a las funciones bibliotecarias automatizadas, mientras que en 1986 era la de adquisiciones la que contaba con un porcentaje mayor en las bibliotecas universitarias, hoy se ve claramente sobrepasada por la catalogación y el catálogo de acceso público.

El hecho de que la catalogación sea la función con el mayor porcentaje de automatización responde al deseo de llegar a conseguir un verdadero sistema de catalogación centralizado/compartido de una manera eficiente, ahorrando, de esta forma, tiempo y trabajo al personal bibliotecario.

Los catálogos de acceso público eran prácticamente inexistentes en 1986 (sólo podian consultarse en dos universidades). Hoy, de las quince universidades estudiadas, trece han incorporado el catálogo en línea. La posibilidad de acceder a la información de una forma no lineal, utilizando, por ejemplo, operadores de Boole $\mathrm{y} / \mathrm{o}$ de proximidad, permite la elaboración de estrategias de búsqueda mucho más precisas que posibilitan una recuperación de la información deseada más acorde con las necesidades reales. Esta gran ventaja de los catálogos en línea, frente a los tradicionales en fichas, es una de las principales razones que influyen en el alto porcentaje de automatización de este servicio.

El catálogo colectivo en línea de publicaciones periódicas de las universidades catalanas era sólo un proyecto en 1986. Hoy es una realidad, aunque sólo puede consultarse desde algunos centros participantes, por lo que su accesibilidad es aún restringida.

El tamaño de los catálogos automatizados en las bibliotecas universitarias ha crecido de modo mucho más rápido en el campo de las monografias que en el de las publicaciones periódicas. Mientras que en las primeras se ha multiplicado aproximadamente por cuatro (de 170.000 registros en 1986 se ha pasado a 697.732 ) en las segundas ni siquiera ha llegado a duplicarse (en 1986 habia 40.500 y hoy hay 59.601). Esto se debe, principalmente, a que las universidades que han automatizado sus bibliotecas con DOBIS/LIBIS no tienen automatizados los registros de publicaciones periódicas, y ya que la mayoria han elegido este sistema no se ha producido un incremento significativo en la automatización de este tipo de fondos. Hay que indicar que todas las cifras referentes a números totales de registros 
incluyen un número de duplicados imposible de conocer al no existir un catálogo colectivo.

En cuanto al atraso que las bibliotecas universitarias llevan en el proceso de conversión retrospectiva, éste es la consecuencia lógica de la falta de infraestructura necesaria para poder llevarlo a cabo; concretamente falta de personal y medios económicos necesarios para ello. Influye también negativamente la escasez de experiencias y estudios que puedan, al menos, guiar a los bibliotecarios en la elección de los métodos más eficaces para realizar uno de los trabajos más lentos y tediosos en lo que a informatización de bibliotecas se refiere.

El alto porcentaje de bibliotecas universitarias que utilizan CD-ROM (para alguna de las funciones descritas anteriormente), es el resultado de las ventajas que este nuevo medio ofrece (4). Es también importante señalar que el CD-ROM está presente en muchas bibliotecas universitarias aun cuando éstas no se encuentren mecanizadas.

Entre los nuevos servicios introducidos en las bibliotecas universitarias desde 1986, son quizás el acceso a bases de datos en línea, la petición automatizada de documentos primarios, y el acceso a catálogos distintos del de la propia biblioteca los que más influyan en una mejora directa del servicio que estos centros proporcionan a sus usuarios.

La necesidad de colaboración entre las bibliotecas universitarias, con objetivos comunes y problemas similares, no había dado todavía fruto en 1987. Hoy existen las redes DOBIS/LIBIS y REBIUN, aunque todavía no podemos hablar de resultados concretos, especialmente en el segundo caso.

\section{Conclusiones}

La tendencia a mecanizar los servicios bibliotecarios responde a dos realidades. Por un lado, la automatización ha demostrado proporcionar un mejor servicio a los usuarios. Por otro, en la actualidad, parece obvio que un adecuado servicio a los usuarios de estos centros impone, sin duda, la utilización de sistemas informáticos.

Sin embargo, aunque el número de universidades españolas que han optado por implantar sistemas informáticos en sus bibliotecas ha crecido considerablemente desde 1986, existe aún un número importante que no lo ha hecho.

Es del todo imposible proporcionar a los usuarios una información actualizada sin recurrir al uso de bases de datos en línea, y, sin embargo, este servicio es todavía incipiente en las universidades españolas. En centros donde la actualidad de la información requerida es de suma importancia, es imprescindible tener acceso a este servicio (la actualización de la información en las bases de datos en línea es la mayor con respecto a la de las bases de datos en CD-ROM y a la de los repertorios impresos). Es por tanto, imprescindible, que este servicio forme parte, lo antes posible, de todas las bibliotecas universitarias.

Sin duda, la falta de acceso a bases de datos en línea intenta suplirse mediante la adquisición de bases de datos en CD-ROM, que va siendo cada vez mayor en este tipo de bibliotecas. Así, bibliotecas universitarias que no están automatizadas, sí cuentan, sin embargo, con productos en CD-ROM. Y a pesar de que la 
utilización de éstos es muy baja comparada con la de otros países europeos (3), es, sin embargo, significativa.

Por otra parte, aun cuando la automatización del servicio de préstamo es quizás una de las menos complicadas de llevar a cabo, y agiliza este proceso considerablemente (hecho más que deseable al tratarse de centros con un número elevado de usuarios), sólo la tercera parte de las bibliotecas universitarias tienen este módulo en funcionamiento.

En cuanto a la situación de la conversión retrospectiva, urge plantearse la necesidad de agilizar este proceso, invirtiendo más tiempo y medios para ello, y realizando mayor número de estudios que investiguen los métodos más eficaces para su realización.

De este estudio se desprende que la automatización de las bibliotecas universitarias se encuentra en una fase intermedia, estando automatizadas un $41 \%$ de las mismas.

Aun cuando no se trata de una situación ideal, es esperanzador constatar cómo, con el paso del tiempo, la situación ha mejorado considerablemente, y cómo el éxito de la implantación de sistemas automatizados en algunas de las bibliotecas universitarias anima al resto a plantearse tal posibilidad.

\section{Notas y Bibliografia}

1. Los autores de este estudio fueron Rosa María Capo y Alice Keefer, por parte de la asociación SOCADI; y José Maria Gómez y Antonio Magariños, por parte de la asociación ANABAD.

2. Es necesario aclarar que estos porcentajes no incluyen un número importante de bibliotecas que utilizan el sistema PALS, en concreto las de la Xarxa de Catalunya, debido a que la información relativa a este tipo de bibliotecas se recibió cuando el plazo de recepción de datos ya se había cerrado. Así, hay que tener en cuenta que el porcentaje de utilización del sistema PALS es, en la realidad, superior al señalado.

3. RAITT, D. I., y CHEN, Ch. Use of CD-ROMs in Spanish Libraries. Revista Española de Documentación Cientifica, 13 (2), 710-716, 1990.

4. MOSCOSO CASTRO, P. y NOUAR, J. Introducción al CD-ROM: Su tecnología y aplicaciones bibliotecarias. Boletín de la $A N A B A D$, XXXIX (2), 1-15, 1990. 